\title{
Apprentissage profond : un troisième oeil pour les praticiens
}

\section{A. Fourcade (Paris)}

" L'intelligence artificielle connaît un essor fulgurant depuis ces dernières années. L'apprentissage automatique et plus précisément l'apprentissage profond grâce aux réseaux de neurones convolutifs ont permis des avancées majeures dans le domaine de la reconnaissance des formes. Cette présentation fait suite à mon travail de thèse. La première partie retrace l'historique et décrit les principes de fonctionnement de ces réseaux. La seconde présente une revue de la littérature de leurs applications dans la pratique médicale de plusieurs spécialités, pour des tâches diagnostiques nécessitant une démarche visuelle (classification d'images et détection de lésions). Quinze articles, évaluant les performances de ces solutions d'automatisation, ont été analysés. La troisième partie est une discussion à propos des perspectives et des limites présentées par les réseaux de neurones convolutifs, ainsi que leurs possibles applications en chirurgie orale.» 